\title{
Metacognition, Self Regulation, and Self-regulated Learning: A Rose by any other Name?
}

\author{
Susanne P. Lajoie
}

Published online: 19 September 2008

(C) Springer Science + Business Media, LLC 2008

\begin{abstract}
This commentary reviews the distinctions researchers make in defining metacognition, self-regulation, and self-regulated learning along with the methods used to explore these constructs. Bandura's notion of reciprocal determinism (1977) is revisited in the context of situated learning, whereby interactions between the person, behavior, and environment take on new meaning when examining learning and affect in specific contexts where knowledge is constructed through interacting with all that the environment affords, be that material or human. The interaction between the mind and environment continues to be an interesting question with regard to these three constructs, and this interaction can be explored by using computers as cognitive tools. Technology-rich environments are described that provide opportunities for assessing and validating metacognition, self-regulation, and self-regulated learning with future directions for assessing co-regulation of teams of learners.
\end{abstract}

Keywords Metacogniton S Self-regulation · Self-regulated learning ·

Computer based learning environments

Many reputable scholars in our field have discussed how theories evolve either through evolutionary or revolutionary methods where the fittest theories, concepts, and constructs predominate the literature and guide research for several years and decades (Kuhn 1996; Mayer 1997). Theory change can be seamless such as a natural progression where old ideas die when they have outlived their usefulness and new ones thrive. On the other hand, theory change can become a battle of words that polarizes our field into different camps. Theory change based on empirical findings and constructive dialogue is healthy. The research presented in this special issue on metacognition, self-regulation (SR), and self-regulated learning (SRL) is unique because it serves to move the field forward without alienating those who have been working within a paradigm with one set of assumptions for several years. The contributors to this journal outline the distinctions between these three constructs and highlight where the edges are blurred. Most importantly, they seek ways to improve our 
understanding of these important constructs by pushing us to consider definitions that can be operationalized so that appropriate methods and assessments can be developed.

Dinsmore et al. (2008) address these issues thoroughly and provide an analysis of 255 studies where metacognition, SR, and SRL have been defined in multiple ways. Their results were illuminating both in terms of the variance in which definitions were proposed and in the types of methods used to assess these constructs. Their analysis provides great insight into how the field defines, or fails to define, these concepts and how methods have been designed, or not designed, to assess them. The result of this analysis demonstrates that the terms metacognition, $S R$, and $S R L$ have often been used interchangeably or in some cases embedded within each other. For the purpose of moving research forward, naming is important, and a rose by any other name does not smell as sweet since it causes confusion when interpreting research findings.

Dinsmore et al. (2008) provide a historical review of how metacognition, SR, and SRL have been defined. The term metacognition is attributed to Flavell (1971) who defined metacognition as thinking about one's own thinking. He described metacognition from a developmental perspective where we learn to monitor our thinking by knowing what to monitor (metacognitive knowledge) which grows out of experience, setting goals to achieve understanding and activating strategies to reach goals and evaluate one's progress.

Metacognitive skills are developed through the interaction of such processes. Selfregulation grew out of this definition when Baker and Brown (1984) separated the metacognitive term into knowledge about cognition (monitoring) and self-regulatory mechanisms for checking the outcome, planning, monitoring effectiveness, testing, revising, and evaluating strategies. The cognitive aspects of SR were expanded upon by Bandura $(1982,1986)$ when he referred to the importance of considering behavioral and emotional regulation in his research on self-efficacy. Bandura (1977) raised the importance of reciprocal determinism where human functioning is based on an interaction between person, behavior, and environment reasoning that SR cannot occur without interaction with the environment.

As self-regulation became a focus in academia, the term SRL emerged (Zimmerman 1989), and Corno and Mandinach (1983) described an integrated theory of SRL that addresses the interaction of cognitive, motivational, and contextual factors. The role of volition in pursuing a topic and in sustaining effortful learning is an aspect of SR and SRL that can be traced to Bandura (1977) and Corno and Mandinach's (1983) early work. The affective dimension of SR and SRL continues to be an important dimension and is beginning to be explored in interesting ways (Boekaerts and Cascallar 2006; Pintrich 2004; Winne and Perry 2000; Witherspoon et al. 2008). Loyens et al. (2008) provide some insights in this regard with respect to the role of affect in their examination of problembased learning and self-directed learning where realistic scenarios can serve to engage learners. Calibrating the relation between affect, such as confidence judgments, and actual process or performance is an area to be explored (Nietfeld and Schraw 2002). Maggioni and Parkinson (2008) point to research that illustrates the complexity of this relation as it pertains to teacher beliefs and calibration with respect to knowledge and experience and their effects on pedagogical practices.

\section{Exploring Reciprocal Determinism from a Situated Learning Perspective}

In the analysis of research of Dinsmore et al. (2008) in the area of metacognition, SR, and SRL they found that $49 \%$ of researchers who used such terms explicitly defined them. 
However, a greater percentage of researchers explicitly defined SRL (69\%), followed by SR $(57 \%)$ and metacognition (32\%). They theorize that these differences may be explained since SRL and SR are often defined within specific contexts, whereas metacognition is a well-established term, and consequently, researchers do not define it as readily. They caution for the need for more explicit definitions so that adequate comparisons and generalizations can be made about these important constructs. They also caution against the sole use of self-report data (which are the measures of choice in $43 \%$ of the articles where SR and SRL were defined).

Winne and Perry (2000) call attention to the fact that there is very little information on what individuals are thinking or doing in many studies in this area, and the consensus is that there is a need for broader and convergent methods to establish multiple forms of evidence of what individuals are doing and thinking about when we say that metacognition, SR, or SRL occurs and how it occurs. In other words, we need better mechanisms that provide a deeper understanding of how monitoring and regulation occurs within specific tasks. Further, monitoring and regulation can also be influenced by emotion and affect, and we must consider these points in exploring new methodologies.

Dinsmore et al. (2008) distinguish between those studying SR and metacognition, suggesting that the former focus on how the environment stimulates the individual's awareness and regulatory response, whereas the latter researchers emphasize that the mind of the individual is the trigger for subsequent judgments or evaluations. SRL distinguishes itself by having a focus on academic learning (Pintrich 2004; Zimmerman 1989) and lately in professional learning contexts such as medicine (Lajoie et al. 2006). In each of these examples, we see evidence of what Bandura (1977) referred to as reciprocal determinism whereby these constructs can be explained through the interaction between the person, behavior, and environment. We have come to see learning as situated within specific contexts and knowledge as constructed through interacting with all that the environment affords, be that material or human resources (Brown et al. 1989; Collins et al. 1989; Greeno 1989; Lave and Wenger 1991). Learning, as suggested by Shepard (2000) is an active process of mental construction and sense making. Once again, it is the interaction between the mind and environment that presents the most interesting questions in terms of the active nature of learning.

\section{Technology-Rich Environments for Extending Metacognition, SR, and SRL Contexts}

Computers as cognitive tools (Jonassen and Reeves 1996; Lajoie 2005; Lajoie and Derry 1993; Salomon et al. 1991) afford the learner richer opportunities for the types of interactions that would support metacogniton, SR, and SRL. In some instances, the computer environment stimulates the mind by providing external constraints or scaffolds to guide learning. In other situations, individuals use cognitive tools to discover new knowledge on their own. Technology-rich environments can be designed with cognitive tools that model human behavior or provide complex simulations that learners can attend to and learn from (Lajoie and Azevedo 2006). Once such models are internalized, learners can deliberately practice the correct skills rather than practice indiscriminately (Ericsson 2002).

Some cognitive tools lead to deeper understanding by supporting cognitive processes, supporting lower-level cognitive processes in order to free up cognitive resources for higher-level thinking skills, reifying student problem solving by providing computer representations of their problem solving efforts, or providing visualization and argumentation environments where students can learn effectively from each other. Cognitive tools 
scaffold learners either by explicit computer tutoring and feedback, through the structure of the learning environment itself or by a combination of computer tools and human tutoring support. Such elements serve to externally regulate an individual's cognition, motivation, or behavior by guiding or scaffolding the individual in terms of what, how, and when to do a task. Such tools can facilitate or constrain individuals' attempts to regulate their learning. Winne (1997) suggested that internal and external feedback is critical to enhancing selfregulation. Internal feedback is produced by the learners' own monitoring processes when they evaluate their performance, whereas external feedback is given by other people or events to the learner. External feedback can inform the learner about domain knowledge errors but can also enhance learners' effective engagement in tasks.

As Winters et al. (2008) point out, computer-based learning environments (CBLEs) provide an exciting context in which to study SRL given that they utilize a range of computer tools, visual, textual, and auditory that can be interwoven to assist individuals in learning for a specific educational purpose (Azevedo 2005). Azevedo demonstrated how different forms of scaffolding can be designed to prompt and cue for SRL.

Having said this, learners eventually need to be able to do things independently (i.e., without assistance). Individuals take the initiative with or without help in diagnosing needs, formulating learning goals, evaluating learning outcomes (Knowles 1975). The research on self-directed learning closely parallels research on self-regulation, revealing the active nature of learning (Nenniger 1999). The intersection between how the environment can stimulate individual awareness and how the mind serves as an initiator for judgments and evaluations needs further exploration. The results of such research can serve to illuminate the mechanisms of metacognition, SR, and SRL that can lead to appropriate instructional interventions to promote thinking and learning.

Before environments can be designed to stimulate individual awareness, researchers need to identify what the focus of such awareness should be on. The expertise literature provides a starting point. Of specific interest to this commentary is that experts across domains consistently score higher on measures of metacognition than their novice counterparts (Chi et al. 1988). Knowing what to attend to in a specific domain is essential for becoming an expert in that domain. Consequently, identifying the cognitive components that learners need to attend to in order to become more expert-like in their performance is step one to developing external models and feedback for novices that can assist them in becoming more proficient.

Multiple measures are used to identify how experts think. One measure in particular is the use of think-alouds that provide a window on how experts think. As Winne (2000) pointed out, a criticism of research in the area of metacogniton, SR, and SRL has been the over-reliance on self-report data without having evidence of what individuals were doing at a particular point in time. He suggested combining think-alouds with other measures to provide a deeper understanding of these constructs. We have been developing converging methods using technology-rich environments to situate learning in ways that facilitate metacognition, SR, and SRL (Gauthier and Lajoie, in preparation; Lajoie et al., in preparation; Lu and Lajoie 2008).

We examine the premise that experts are more aware of their own thinking and are aware of what they know or do not know in the context of medical case-based teaching (Gauthier and Lajoie, in preparation). Medical case-based instructors often teach by performing thinkalouds of how they would solve a patient case and thereby externalize their thoughts for the social purpose of instruction. Teachers perform these think-alouds early in the instructional sessions to model their reasoning and expectations for students. As with all cognitive apprenticeships, this explicit modeling fades as students acquire new knowledge and then 
the teacher facilitates and guides the learning process by asking open-ended general questions that encourage students to think about what they have done and why (HmeloSilver and Barrows 2006; Lajoie et al. 2001). Feedback and reflection on the learning process are essential components of PBL and cognitive apprenticeship (Loyens et al. 2008; Williams 1992). Analyzing the content of medical pedagogical interactions can help determine what models and feedback are most conducive to learning and ultimately lead to more effective practice.

Given the natural tendency of medical instructors to externalize their thoughts, we asked them to think or teach out loud while they solved a set of standardized patient cases using a computer-based learning environment, BioWorld (Lajoie 2005). A mixed-method approach was used to capture, represent, and validate case-specific solutions. Converging sources of data were used to develop an accurate interpretation of expert clinical problem solving in these situations, as well as on their metacognitive processes (i.e., planning, monitoring, control, and reflection). The protocol analyses were combined with computer logs that situated the verbal protocols in terms of actual actions taken while solving cases. These converging forms of evidence were interpreted and used to design multilayered visual representations of the problem-solving processes for each case.

Experts were asked to reflect on their own problem solving by reviewing the multilayered case solution. This review process was a form of self-assessment, since participants could reflect on what they said, validate their own problem solving, and modify things that needed to be changed. We analyzed what instructors, do, say, and modify once they reflect on their own solutions. The results of these analyses provide us with a rich description of what expert medical instructors attend to, and are aware of, during and after their case-based instruction. These findings provide a roadmap for designing scaffolding tools that can help novices become aware of salient aspects of medical problem solving.

Another area for exploration and expansion is the area of co-regulation. In other words, can the group that one works with help regulate the learning experience? We have started to look at this in the context of simulated emergencies that mimic the types of decisionmaking that is needed by medical teams in life-threatening situations. More specifically, we investigated collaborative decision making in this context as well as the communicative discourse of groups of learners engaged in a simulated medical emergency (Lu and Lajoie 2008). We examined the complex interactions that occur during the activity that promote learning, co-regulation, and ultimately self-regulation. Traditionally, the instructor would use a whiteboard to document the medical argument as it evolved throughout the simulation (we call this the traditional whiteboard condition, TW). We introduced a second condition using an interactive whiteboard (IW, EBeam technology) to see whether or not collaborative decision-making would be enhanced.

Our assumption was that the IW would facilitate communication beyond the teacherstudent, to include student-student both within and between the various subgroups. The IW group could document their medical arguments by using a structured template for constructing, annotating, and sharing arguments. The IW condition differed from the TW condition in that participants engaged in more adaptive decision-making behavior early on in the intervention. This early engagement led to shared understandings and subsequently to more effective patient management. Group differences were also found in the types of collaborative discourse, and the IW groups produced more productive argumentation. These data provide insight into how group discourse and argumentation can be enhanced with tools that support co-regulation. Future research in this area will look at specific indicators in the discourse that operationalize the concept of co-regulation. 


\section{Metacognition, SR, and SRL: Avoiding the Star Crossed Lover Trap}

We began this commentary examining the conclusions of Dinsmore et al. (2008) about how researchers have chosen to define and operationalize metacognition, SR, and SRL. They point out the misuse of terms and definitively point out that these terms are not identical, and thus, a rose by any other name does not smell as sweet. Carrying the Romeo and Juliet metaphor one step further, we do not want misconceptions to lead to death. This special issue points to ways in which research can take on new life through better definitions, more robust methodologies and new environments such as CBLEs or PBLS that can lead to new ways of understanding metacognition, SR, and SRL.

\section{References}

Azevedo, R. (2005). Computer environments as metacognitive tools for enhancing learning. Educational Psychologist, 40, 193-197. doi:10.1207/s15326985ep4004_1.

Baker, L., \& Brown, A. L. (1984). Metacognitive skills and reading. In P. D. Pearson (Ed.), Handbook of reading research (pp. 353-394). New York: Longman.

Bandura, A. (1977). Social learning theory. Oxford, England: Prentice-Hall.

Bandura, A. (1982). Self-efficacy mechanism in human agency. The American Psychologist, 37, $122-147$. doi:10.1037/0003-066X.37.2.122.

Bandura, A. (1986). Social foundations of thought and action: A social cognitive theory. Englewood Cliffs, NJ: Prentice-Hall.

Boekaerts, M., \& Cascallar, E. (2006). How far have we moved toward the integration of theory and practice in self-regulation? Educational Psychology Review, 18, 199-210. doi:10.1007/s10648-006-9013-4.

Brown, J. S., Collins, A., \& Duguid, P. (1989). Situated cognition and the culture of learning. Educational Researcher, 18, 32-42.

Chi, M. T. H., Glaser, R., \& Farr, M. J. (1988). The nature of expertise pp. ix-xvii. Hillsdale, NJ: Erlbaum.

Collins, A., Brown, J. S., \& Newman, S. E. (1989). Cognitive apprenticeship: Teaching the craft of reading, writing, and mathematics. In L. B. Resnick (Ed.), Knowing, learning, and instruction: Essays in honor of Robert Glaser (pp. 453-494). Hillsdale, NJ: Erlbaum.

Corno, L., \& Mandinach, E. B. (1983). The role of cognitive engagement in classroom learning and motivation. Educational Psychologist, 18(2), 88-108.

Dinsmore, D. L., Alexander, P. A., \& Loughlin, S. M. (2008). Focusing the conceptual lens on metacognition, self-regulation, and self-regulated learning. Educational Psychology Review (this issue).

Ericsson, K. A. (2002). Attaining excellence through deliberate practice: Insights from the study of expert performance. In M. Ferrari (Ed.), The pursuit of excellence in education (pp. 21-55). Hillsdale, NJ: Erlbaum.

Flavell, J. H. (1971). First discussant's comments: what is memory development the development of? Human Development, 14, 272-278.

Greeno, J. (1989). A perspective on thinking. The American Psychologist, 44, 134-141. doi:10.1037/0003066X.44.2.134.

Hmelo-Silver, C. E., \& Barrows, H. S. (2006). Goals and strategies of a problem-based learning facilitator. Interdisciplinary Journal of Problem-based Learning, 1(1), 21-39.

Jonassen, D. H., \& Reeves, T. C. (1996). Learning with technology: Using computers as cognitive tools. In D. H. Jonassen (Ed.), Handbook of research for educational communications and technology (pp. 693-719). New York: Simon \& Schuster.

Knowles, M. S. (1975). Self-directed learning: A guide for learners and teachers. Englewood Cliffs, NJ: Prentice Hall.

Kuhn, T. S. (1996). The structure of scientific revolutions (3rd ed.). Chicago: The University of Chicago Press.

Lajoie, S. P. (2005). Cognitive tools for the mind: The promises of technology: Cognitive amplifiers or bionic prosthetics? In R. J. Sternberg, \& D. Preiss (Eds.), Intelligence and technology: Impact of tools on the nature and development of human skills (pp. 87-102). Mahwah, NJ: Erlbaum.

Lajoie, S. P., \& Azevedo, (2006). Teaching and learning in technology-rich environments. In P. A. Alexander, \& P. H. Winne (Eds.), Handbook of educational psychology (pp. 803-821, 2nd ed.). Mahwah, NJ: Erlbaum.

Lajoie, S. P., \& Derry, S. J. (Eds.).(1993). Computers as cognitive tools. Hillsdale, NJ: Erlbaum. 
Lajoie, S. P., Faremo, S., \& Wiseman, J. (2001). Identifying human tutoring strategies for effective instruction in internal medicine. International Journal of Artificial Intelligence in Education, 12(3), 293309 .

Lajoie, S. P., Faremo, S., Wiseman, J., \& Gauthier, G. (2006). Promoting self-regulation in medical students through the use of technology. Technology Instruction Cognition and Computing, 3(1-2), 81-87.

Lave, J., \& Wenger, E. (1991). Situated learning. Legitimate peripheral participation. Cambridge: University of Cambridge Press.

Loyens, S. M. M., Magda, J., \& Rikers, M. J. P. Self-directed learning in problem-based learning and its relationships with self-regulated learning. Educational Psychology Review (this issue).

Lu, J., \& Lajoie, S. P. (2008). Supporting medical decision making with argumentation tools. Contemporary Educational Psychology, 33, 425-442. doi:10.1016/j.cedpsych.2008.05.005.

Maggioni, L., \& Parkinson, M. M. The role of teacher epistemic cognition, epistemic beliefs, and calibration in instruction. Educational Psychology Review (this issue).

Mayer, R. E. (1997). Learners as information processors: legacies and limitations of educational psychologies second metaphor. Educational Psychologist, 31(3/4), 151-161.

Nenniger, P. (1999). On the role of motivation in self-directed learning. The 'two-shells-model of motivated self-directed learning' as a structural explanatory concept. European Journal of Psychology of Education, 14(1), 71-86.

Nietfeld, J. L., \& Schraw, G. (2002). The effect of knowledge and strategy training on monitoring accuracy. The Journal of Educational Research, 95(3), 131-142.

Pintrich, P. R. (2004). A conceptual framework for assessing motivation and self-regulated learning in college students. Educational Psychology Review, 16, 385-407.

Salomon, G., Perkins, D. N., \& Globerson, T. (1991). Partners in cognition: extending human intelligence with intelligent technologies. Educational Researcher, 20(3), 2-9.

Shepard, L. A. (2000). The role of assessment in a learning culture. Educational Researcher, 29(7), 4-14.

Williams, S. M. (1992). Putting case-based instruction into context: Examples from legal and medical education. Journal of the Learning Sciences, 2(4), 367-427. doi:10.1207/s15327809jls0204_2.

Winne, P. H. (1997). Experimenting to bootstrap self-regulated learning. Journal of Educational Psychology, 89, 397-410. doi:10.1037/0022-0663.89.3.397.

Winne, P. H., \& Perry, N. E. (2000). Measuring self-regulated learning. In M. Boekaerts, P. R. Pintrich, \& M. Zeidner (Eds.), Handbook of self-regulation (pp. 531-566). San Diego, CA: Academic Press.

Winters, F. I., Greene, J. A., \& Costich, C. M. (2008). Self-regulation of learning within computer-based learning environments: A critical analysis. Educational Psychology Review.

Witherspoon, A. M., Azevedo, R., \& D’Mello, S. (2008). The dynamics of self-regulatory processes within self-and externally regulated learning episodes during complex science learning with hypermedia. In B. Woolf, E. Aimeur, R. Nkambou, \& S. P. Lajoie (Eds.), Intelligent tutoring systems: 9th International Conference on Intelligent Tutoring Systems, ITS 2008 (pp. 260-269). New-York: Springer-Verlag.

Zimmerman, B. J. (1989). A social cognitive view of self-regulated academic learning. Journal of Educational Psychology, 81, 329-339. doi:10.1037/0022-0663.81.3.329. 